\title{
Mycoplasma genitalium and Mycoplasma hominis infection in south Korea during 2018-2020
}

\author{
Eun Ju Oh ${ }^{1 \neq}$, Tae Su Jang ${ }^{2 *}$, Jae Kyung Kim ${ }^{3 *}$ \\ ${ }^{I}$ Departmentof Medical Laser, School of Medicine, Dankook University Graduate, Chungnam, South Korea \\ ${ }^{2}$ Department of Preventive Medicine, Dankook University College of Medicine, Chungnam, South Korea \\ ${ }^{3}$ Departmentof Biomedical Laboratory Science, Dankook University College of Health Sciences, Chungnam,
} South Korea

Received: July 2021, Accepted: September 2021

\begin{abstract}
Background and Objectives: Sexually transmitted infections (STIs) can remain undetected and untreated; therefore, rapid diagnosis and treatment of STIs are important. Mycoplasma genitalium (MG), Mycoplasma hominis (MH), and Ureaplasma urealyticum are sexually transmitted pathogens that cause asymptomatic, organ-specific, and chronic infections, thereby posing a threat to community health. Therefore, we investigated the epidemiological trends of MG and MH infections in South Korea for rapid diagnosis and treatment.

Materials and Methods: From September 2018 to December 2020, samples (catheter, pus, tissue, swab, and urine) were collected from outpatients of hospitals in South Korea for molecular biological venereal disease testing. DNA was extracted and analyzed using real-time polymerase chain reaction.

Results: Of the 59,381 samples analyzed, 8.78\% (n=5,215) were positive for MG and MH. The MH positivity rate $(5.51 \%$, $\mathrm{n}=3,273)$ was higher than the MG positivity rate $(3.27 \%, \mathrm{n}=1,942)$. MG and MH positivity rates were the highest in patients aged $<19$ years. Men had higher MG positivity rate, whereas women had higher MH positivity rates. Furthermore, the MGpositivity rate was the highest in the swab samples of both men and women, whereas that of MH was the highest in the urine samples of men and swab samples of women.

Conclusion: We identified the differences between MG and MH positivity rates based on sex, specimen, and age. Our findings can provide information for strategies that protect public health and reduce STI incidence and transmission.
\end{abstract}

Keywords: Sexually transmitted infection; Mycoplasma genitalium; Mycoplasma hominis; Infection

\section{INTRODUCTION}

Sexually transmitted infections (STIs) are common life-threatening infectious diseases transmitted through sexual contact between people (1). The World Health Organization estimates that over 1 million STIs are contracted globally every day (2). Furthermore, the Centers for Disease Control and
Prevention of the United States estimated that in 2018, approximately $20 \%$ of the US population had an STI on any given day, and STIs cost the healthcare system nearly $\$ 16$ billion (3).

Because STIs often cause asymptomatic or nonspecific symptoms, which remain untreated and ultimately lead to morbidity and mortality, rapid diagnosis and treatment of STIs are important (4). In

"Corresponding author: Jae-Kyung Kim, Ph.D, Departmentof Biomedical Laboratory Science, Dankook University College of Health Sciences, Chungnam, South Korea. Tel: +82-415501451_Fax: +82-415597934Ｅmail: nerowolf2@dankook.ac.kr

${ }^{¥}$ These authors contributed equally to this project and should be considered co-first authors.

Copyright $\odot 2021$ The Authors. Published by Tehran University of Medical Sciences.

(c) (7) This work is licensed under a Creative Commons Attribution-Non Commercial 4.0 International license

(https://creativecommons.org/licenses/by-nc/4.0/). Noncommercial uses of the work are permitted, provided the original work is properly cited. 
addition, STIs can increase the infectivity of HIV; thus, regular testing for STIs is important (5).

STIs are caused by a wide range of pathogens, including bacteria, viruses, protozoa, and fungi. The major causative pathogens include Ureaplasma urealyticum (UU), Mycoplasma hominis (MH), Chlamydia trachomatis (CT), Neisseria gonorrhoeae (NG), M. genitalium (MG), and Trichomonas vaginalis (TV) (6).

Urogenital tract infection has become a major concern among sexually active individuals worldwide. Therefore, in its analysis of treatable diseases in women, the World Health Organization ranked STIs as the second most important condition after cancer (7). In general, MH, MG, and UU are important sexually transmitted bacterial pathogens that can cause asymptomatic, organ-specific, and chronic infections; therefore, they are considered to pose a threat to community health. MG and MH are common pathogens that cause infections in the male and female reproductive systems (8).

MG causes urethritis in men and urethritis and cervicitis in women; a missed or delayed diagnosis and treatment of these infections can also lead to infertility (9). MG may also cause infections in pregnant women and newborns, and there is accumulating evidence on the presence of MG in the cervical mucosa of women (6). A recent meta-analysis by Wiesenfeld et al. (10) identified studies published between June 25, 2014, and December 31, 2016, in PubMed using a single search term, Mycoplasma genitalium. The authors reported that women with MG infection have an approximately two-fold increased risk of cervical inflammation, pelvic inflammation, pre-term delivery, spontaneous abortion, and infertility (10). The incidence and prevalence of MG infections are affected by geographic location and socioeconomic status (11).

$\mathrm{MH}$ is another pathogen commonly identified in the female lower genital tract, particularly in the upper genital organs, that causes genetic diseases (12). Prenatal transmission of $\mathrm{MH}$ results in neonatal death (12). There is also accumulating evidence on the association of $\mathrm{MH}$ with natural abortion, congenital pneumonia, and meningitis (12).

MG and $\mathrm{MH}$ are part of the normal flora in healthy adult male and female urinary reproductive systems; however, they act as opportunistic infectious agents and can cause various complications (13). Therefore, infections caused by these pathogens have clinical importance and require physicians' attention. Studies on their detection rates and detection methods are ongoing worldwide (13).

This study aimed to investigate the epidemiological trends of MG and MH infections in South Korea from September 2018 to December 2020. Our findings can provide information that could be used to protect public health and reduce the incidence and transmission of STIs.

\section{MATERIALS AND METHODS}

Sample collection. Various clinical specimens were collected from male and female outpatients aged $0-\geq 70$ years who visited primary and secondary hospitals in South Korea between September 2018 and December 2020. The specimens were categorized as follows: swab, urine, and other (catheter, pus). The U2 Bio Laboratory (Seoul, Korea) performed the molecular analysis of the biological specimens.

Urine samples were collected from a single urination and stored in containers without preservatives. Briefly, $10 \mathrm{ml}$ of urine was centrifuged at 3,000 rpm for $20 \mathrm{~min}$. The supernatant was discarded, and cell pellets were collected for nucleic acid extraction. The swab samples from the vagina, cervix, and urethral duct were centrifuged at 13,000 rpm for $5 \mathrm{~min}$. Nucleic acids were extracted from the cell pellets after discarding the supernatant. The collected clinical specimens were stored at $-70^{\circ} \mathrm{C}$ until further analysis.

Ethical approval. The study protocol was approved by Dankook University Institutional Review Board (IRB approval number2021-03-056). The study was conducted in conformance with the tenets of the Declaration of Helsinki. Patient consent was waived because this study used statistics from tests conducted by medical institutions for diagnosis and did not use patients' personal information.

Nucleic acid extraction and real-time PCR analysis. Within 48 hours of sample collection, DNA was extracted from the samples using the ExiPrep ${ }^{\mathrm{TM}}$ Dx Bacteria Genomic DNA Kit (Bioneer, Daejeon, South Korea) according to the manufacturer's instructions. Real-time polymerase chain reaction (PCR) assays were performed using the AccuPower ${ }^{\circledR}$ STI8A-Plex Real-Time PCR Kit (Bioneer, Daejeon, South Korea) and AccuPower ${ }^{\circledR}$ STI8B-Plex Real-Time PCR Kit 
(Bioneer, Daejeon, South Korea) on an Exicycler ${ }^{\mathrm{TM}}$ 96 Real-Time Quantitative Thermal Block (Bioneer, Daejeon, South Korea) according to the manufacturer's protocol. Undiluted DNA samples (>20 $\mu \mathrm{g} / \mathrm{ml})$ were quantified using a spectrophotometer (NanoDrop 1000;Thermo Fisher Scientific, USA).

The amplification protocol included one cycle at $95^{\circ} \mathrm{C}$ for $5 \mathrm{~min}$ and 45 cycles at $95^{\circ} \mathrm{C}$ for $5 \mathrm{~s}$ and $55^{\circ} \mathrm{C}$ for $5 \mathrm{~s}$. The cycle threshold value was determined according to manufacturer's instructions. The Limit of detection of MH of AccuPower ${ }^{\circledR}$ STI8B-Plex Real-Time PCR Kit is 269.2 copies/ml, and Limit of detection of MG of AccuPower ${ }^{\circledR}$ STI8A-Plex Real-Time PCR Kit is 333.1 copies/ml. Positive Control DNA concentrations are $3.85 \times 10^{5}, 3.85 \times 10^{4}$, and $3.85 \times 10^{3}$ copies $/ \mathrm{ml}$. The presence of the following 8 pathogens was evaluated: CT, Neisseria gonorrhoeae, UU, MG, TV, MH, herpes simplex virus (HSV)-1, and HSV-2. In this study, we focused on MG and MH. The real-time PCR target genes used and their product sizes are listed in Table 1.

Table 1. Target genes used for real-time PCR analysis and their product sizes.

\begin{tabular}{lcc}
\hline Pathogen & Target genes & Product size (bp) \\
\hline Mycoplasma genitalium & MaPa Gap & 131 \\
Mycoplasma hominis & & 88 \\
\hline
\end{tabular}

Statistical analysis. SAS version 9.4 (SAS Institute Inc., Cary, NC, USA) was used to perform all statistical analyses, including descriptive statistical analysis, frequency analysis. MH and MG DNA detected using real-time PCR were analyzed based on the sex and age of the patients and the type of specimen. A Pvalue $<0.05$ was considered significant.

\section{RESULTS}

In total, 59,381 specimens were tested for the presence of MG and $\mathrm{MH}$ infections during the study period (between August 2018 and December 2020), of which, $8.78 \%(n=5,215)$ tested positive for these pathogens. In addition, the $\mathrm{MH}$ positivity rate (5.51\%, $\mathrm{n}=3,273)$ was higher than the MG positivity rate $(3.27 \%, n=1,942)$. Of the 5,215 samples that tested positive for MG and/or MH, 2.82\% ( $\mathrm{n}=147)$ were cases of MG and MH co-infection.

According to the age-specific analysis, the highest
MG positivity rate was observed in patients aged $<19$ years $(5.95 \%, 61 / 1,026)$, followed by those aged 20 29 years $(5.62 \%, 904 / 16,076)$, whereas the MG lowest positivity rate was observed in patients aged $>70$ years $(0.08 \%, 2 / 2,370)$ (Fig. 1). On the other hand, the $\mathrm{MH}$ highest positivity rate was noted in patients aged $<19$ years $(8.38 \%, 86 / 1,026)$, followed by those aged $20-29$ years $(6.34 \%, 1019 / 16,076)$, whereas the lowest $\mathrm{MH}$ positivity rate was noted in patients aged 40-49 years (2.19\%, 52/11,119) (Fig. 2). Both MG and $\mathrm{MH}$ positivity rates were not significantly associated with age ( $\mathrm{P}=0.066$ and 0.063 , respectively; Table 2 ). However, a distinct infection pattern was observed according to age group.

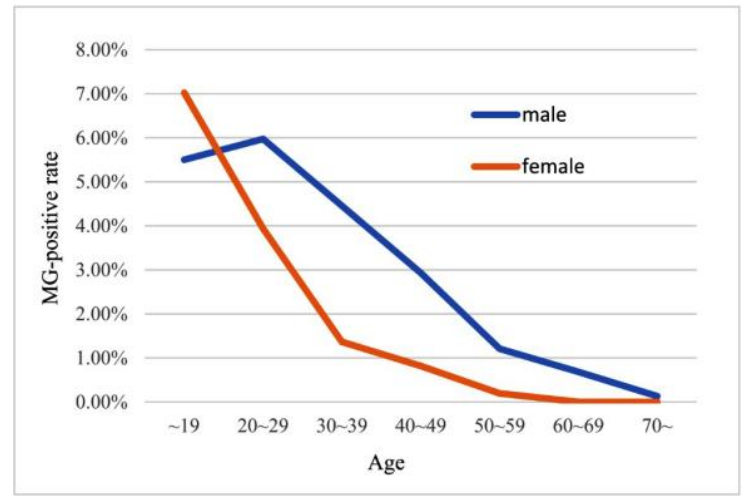

Fig. 1. MGpositivityrate according to ageandsex. The blue line represents MG positivity rates in men. The orange line represents MG positivity rates in women. The highest positivity rates were $5.62 \%(\mathrm{n}=795)$ in men aged 20-29 years and $7.02 \%(\mathrm{n}=21)$ in women aged <19 years.MG: Mycoplasma genitalium.

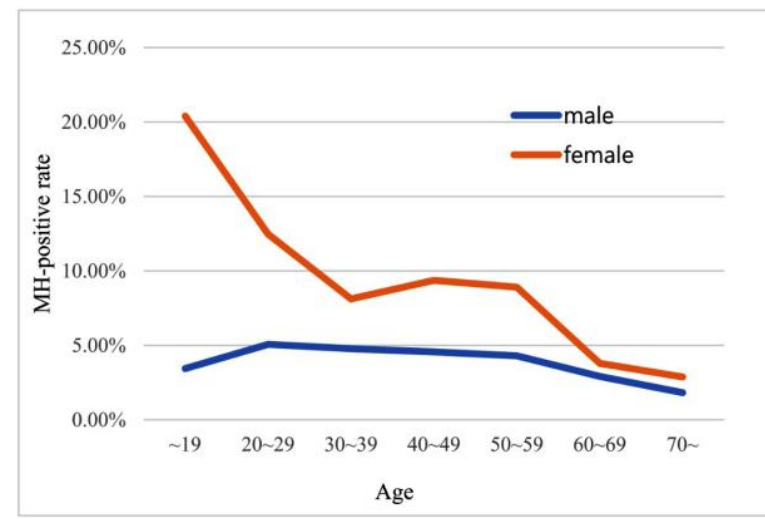

Fig. 2. MH positivityrate according to ageandsex. The blue line represents MH positivity rates in men. The orange line represents $\mathrm{MH}$ positivity rates in women. The highest positivity rates were $5.06 \%(\mathrm{n}=674)$ in men aged $20-29$ years and $20.40 \%$ ( $\mathrm{n}=61)$ in women aged <19 years.MH: Mycoplasma hominis. 
Table 2. Age distribution of MG and MH infection rates

\begin{tabular}{lcclcc}
\hline \multirow{2}{*}{ Age } & \multicolumn{2}{c}{ MG } & & \multicolumn{2}{c}{ MH } \\
\cline { 2 - 3 } \cline { 6 - 7 } & No. & \% & & No. & \% \\
\hline$<19$ & 61 & 5.95 & & 86 & 8.38 \\
$20-29$ & 904 & 5.62 & & 1019 & 6.34 \\
$30-39$ & 611 & 3.91 & & 840 & 5.37 \\
$40-49$ & 266 & 2.39 & & 643 & 5.78 \\
$50-59$ & 76 & 0.90 & & 484 & 5.71 \\
$60-69$ & 22 & 0.47 & & 149 & 3.19 \\
$\geq 70$ & 2 & 0.08 & & 52 & 2.19 \\
$P$ value & & 0.066 & & 0.063 \\
\hline
\end{tabular}

According to the specimen type-specific analysis (Table 3, Fig. 3), the highest MG positivity rate in men was observed in swab specimens $(3.95 \%$, 30/760), and the highest MH positivity rate was observed in urine specimens $(4.59 \%, 1787 / 38905)$. In women, both the highest $\mathrm{MG}$ and $\mathrm{MH}$ positivity rates were noted in swab specimens $(2.16 \%, 144 / 6665$ and $12.29 \%$, 819/6665, respectively). Furthermore, the highest MG positivity rate was observed in the swab samples of men and women. In contrast, the highest $\mathrm{MH}$ positivity rate was noted in the urine samples of men and swab samplesof women.

According to the sex-specific analysis (Table 3), the MG and $\mathrm{MH}$ positivity rates in men were $3.81 \%$ $(1746 / 45,833)$ and $4.51 \%(2,067 / 45,833)$, respectively. In women, the MG and MH positivity rates were $1.45 \%(196 / 13,548)$ and $8.90 \%(1,206 / 13,548)$, respectively. Lastly, MG infections were more prevalent in men, whereas $\mathrm{MH}$ infections were more prevalent in women.

\section{DISCUSSION}

In this study, we investigated the epidemiological trends of MG and MH infections in Korea from Sep-

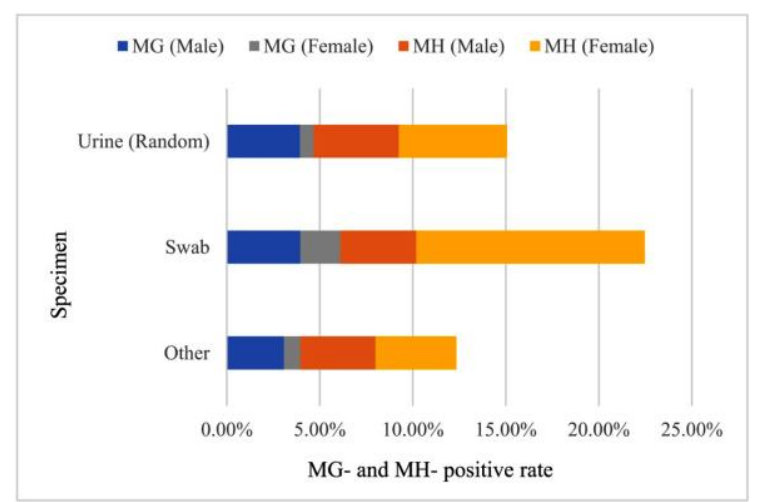

Fig. 3. Positivity rates of MG and $\mathrm{MH}$ infections according to the specimen type and sex. The highest MG positivity rates from swab specimens were $3.95 \%(n=30)$ in men and $2.16 \%(n=144)$ in women. The highest MH positivity rates in men was $4.59 \%(n=1787)$ in urine specimens and $12.29 \%(n=819)$ in swab specimens in women. The blue and gray bars represent MG positivity rates in men and women, respectively. The orange and yellow bars represent $\mathrm{MH}$ positivity rates in men and women, respectively.

tember 2018 to December 2020 and found MG and $\mathrm{MH}$ positivity rates vary according to age, gender, and specimen type.

Baumann et al. conducted large nationally representative surveys in highly developed countries (i.e., Denmark, Great Britain, Norway, Russian Federation, and the United States). They reported that the average prevalence rate of MG was $1.3 \%$ (95\% confidence interval $1.0 \%-1.8 \%$, three studies, $\mathrm{I}^{2}: 41.5 \%$ ) in adults aged $16-44$ years, and that there was no significant difference in MG prevalence between men and women ( $P=0.47$ ) (16). Similarly, Sally et al. found no significant associations between MG and $\mathrm{MH}$ prevalence and sociodemographic variables, such as age, marital status, and educational level of the participants (17). However, they noted changes in MG and MH positivity rates across age groups. The highest MG and MH positivity rates were observed in pa-

Table 3. Positivity rates of MG and MH infections according to specimen type and sex.

\begin{tabular}{|c|c|c|c|c|c|c|c|c|c|c|}
\hline \multirow{3}{*}{ Specimen } & \multirow{3}{*}{$\begin{array}{l}\text { Total } \\
\text { (No.) }\end{array}$} & \multicolumn{4}{|c|}{ Male } & \multirow{3}{*}{$\begin{array}{l}\text { Total } \\
\text { (No) }\end{array}$} & \multicolumn{4}{|c|}{ Female } \\
\hline & & \multicolumn{2}{|c|}{ MG } & \multicolumn{2}{|c|}{ MH } & & \multicolumn{2}{|c|}{ MG } & \multicolumn{2}{|c|}{ MH } \\
\hline & & No. & $\%$ & No. & $\%$ & & No. & $\%$ & No. & $\%$ \\
\hline Other* & 6,168 & 189 & 3.06 & 248 & 4.04 & 897 & 8 & 0.89 & 39 & 4.35 \\
\hline Swab & 760 & 30 & 3.95 & 31 & 4.08 & 6,665 & 144 & 2.16 & 819 & 12.29 \\
\hline Urine & 38,905 & 1,527 & 3.92 & 1787 & 4.59 & 5,986 & 44 & 0.74 & 348 & 5.81 \\
\hline Total & 45,833 & 1,746 & 3.81 & 2,067 & 4.51 & 13,548 & 196 & 1.45 & 1,206 & 8.90 \\
\hline
\end{tabular}

MG: Mycoplasma genitalium; MH: Mycoplasma hominis.

*Other includes catheter and pus samples. 
tients aged <19 years, whereas the lowest MG and $\mathrm{MH}$ positivity rates were observed in patients aged $<70$ years and patients aged 40-49 years, respectively. In contrast, Horner et al. and Harold et al. reported that MG infection rates are higher in men than in women, which is in agreement with our results (10, 11). Moridi et al. detected a lower rate of MH infections in the male urethra than in the female urethra in an Iranian population (18). In our study, Swab samples had the highest MG positivity rates in men and women, and men had a higher MG positivity rate and a lower MH positivity rate.

Genital mycoplasma infections have recently been identified as STIs and have attained similar importance as gonorrhea, syphilis, and CT infections (14). According to the Korea Centers for Disease Control (15), the total number of reported STI cases decreased from 24,583 in 2002 to 9,387 in 2006. Thereafter, the number of cases continued to decrease, falling from 8,983 in 2007 to 7,422 in 2010. However, this downward trend ended in 2011, after which the number of patients continued to increase, from 9,238 in 2012 to 38,057 in 2020 . These changes are similar to the patterns we have noted.

This study had some limitations. First, because 3 years can be considered relatively short, our results may be skewed. Second, the geographical location could not be determined because the sample data were anonymously processed, and the patient's residential location could not be determined. Third, in addition to the pathogenic nature of the viral infection, pathogenic epidemics are largely influenced by geographic, climatic, and socioeconomic factors $(19,20)$. Moreover, the proportion of cases of MG infection varies according to geography and socioeconomic status (11). As such, in this study, the detection rates of MG and MH differed according to sex. However, further studies are needed to determine the prevalence of MG and MH based on regional differences.

Despite these limitations, we found that the detection rates of MG and $\mathrm{MH}$ were the highest in participants aged $<19$ years, does this mean that those aged $<19$ years should be specifically targeted for information campaign on $\mathrm{MG}$ and $\mathrm{MH}$ and provides insights into the patterns of MG and MH positivity rates.

\section{CONCLUSION}

MG and $\mathrm{MH}$ are important bacterial pathogens that cause chronic and asymptomatic genital infections. STIs potentially cause several diseases and prevalence vary according to sex and age. Our study findings could aid in devising strategies to protect public health and reduce the incidence and transmission of STIs. In addition, they provide basic data for conducting further STI's clinical research. Therefore, it is necessary to conduct studies on the long-term analysis data of STIs based on geographic region, age, and sex.

\section{REFERENCES}

1. Chung HS, Lee M. Comparative evaluation of multiplex real-time PCR assays for six pathogens of sexually transmitted infections. Ann Clin Microbiol 2017; 20: $1-6$.

2. World Health Organization. Sexually transmitted infections (STIs). 2019. http://www.who.int/mediacentre/factsheets/fs110/en/ (Accessed 14 Jun 2019)

3. Centers for Disease Control and Prevention. Sexually transmitted disease (STDs). 2021. https://www.cdc.gov/std/defaulthtm (Accessed 25 Jan 2021)

4. Rezaei keykhaei K, Rajabpour Nikoo N, Vaez H, Rezaei keikhaei L, Shirazi M, Ghaemi M, et al. Investigating the prevalence of Mycoplasma genitalium and Mycoplasma hominis among women with vaginal infection in Zabol in 2017. J Obstet Gynecol Cancer Res 2019; 4: 141-145.

5. Choe HS, Lee DS, Lee SJ, Hong SH, Park DC, Lee MK, et al. Performance of Anyplex ${ }^{\mathrm{TM}}$ II multiplex real-time PCR for the diagnosis of seven sexually transmitted infections: comparison with currently available methods. Int J Infect Dis 2013; 17: e1134-e1140.

6. Park JO, Jeon JS, Kim JW, Kim JK. Epidemiological trends of sexually transmitted infections among women in Cheonan, South Korea, 2011-2017. Microbiol Biotechnol Lett 2018; 46: 85-90.

7. Yamazaki T, Matsumoto M, Matsuo J, Abe K, Minami $\mathrm{K}$, Yamaguchi H. Frequency of Chlamydia trachomatis in Ureaplasma-positive healthy women attending their first prenatal visit in a community hospital in Sapporo, Japan. BMC Infect Dis 2012; 12: 82.

8. Moridi K, Ghazvini K, Hemmaty M, Hoseiniun H, Torkaman M, Fallah Mehrabadi MH. Prevalence determination of M. hominis and M. genitalium in the semen samples in the northeast of Iran using culture and multiplex polymerase chain reaction. Arch Razi Inst 2021; 76: 41-49.

9. Fookes MC, Hadfield J, Harris S, Parmar S, Unemo 
M, Jensen JS, et al. Mycoplasma genitalium: whole genome sequence analysis, recombination and population structure. BMC Genomics 2017; 18: 993.

10. Wiesenfeld HC, Manhart LE. Mycoplasma genitalium in women: current knowledge and research priorities for this recently emerged pathogen. J Infect Dis 2017; 216(suppl_2): S389-S395.

11. Horner PJ, Martin DH. Mycoplasma genitalium infection in men. J Infect Dis 2017; 216 (Suppl_2): S396-S405.

12. Ng KF, Kee Tan K, Chok MC, Zamil Mohd Muzzamil N, Choo P, Paramasivam U, et al. Mycoplasma hominis, Ureaplasma urealyticum and Chlamydia trachomatis respiratory colonization in Malaysian preterm infants. J Trop Pediatr 2017; 63: 447-453.

13. Yu N, Lee MK. Clinical implications of multiplex PCR detection of fastidious microorganisms in vaginitis patients. Korean J Clin Microbiol 2011; 14: 30-35.

14. Jeon JS, Kim JK. Epidemiological trends of sexually transmitted infections with Ureaplasma urealyticum among women in Cheonan, South Korea: 2006-2017. Microbiol Biotechnol Lett 2018; 46: 425-429.

15. Korea Disease Control and Prevention Agency. Infectious disease portal. 2021. http://www.kdca.go.kr/npt/biz/npp/iss/stisStatisticsMai n.do (Accessed22 Jun 2021).

16. Baumann L, Cina M, Egli-Gany D, Goutaki M, Hal- beisen FS, Lohrer GR, et al. Prevalence of Mycoplasma genitalium in different population groups: systematic review and meta-analysis. Sex Transm Infect 2018; 94 : 255-262.

17. Adebamowo SN, Ma B, Zella D, Famooto A, Ravel J, Adebamowo C, et al. Mycoplasma hominis and Mycoplasma genitalium in the vaginal microbiota and persistent high-risk human papillomavirus infection. Front Public Health 2017; 5: 140.

18. Moridi K, Hemmaty M, Azimian A, Fallah MH, Khaneghahi Abyaneh H, Ghazvini K. Epidemiology of genital infections caused by Mycoplasma hominis, $M$. genitalium and Ureaplasma urealyticum in Iran; a systematic review and meta-analysis study (2000-2019). BMC Public Health 2020; 20: 1020.

19. Liu WK, Chen DH, Tan WP, Qiu SY, Xu D, Zhang L, et al. Paramyxoviruses respiratory syncytial virus, parainfluenza virus, and human metapneumovirus infection in pediatric hospitalized patients and climate correlation in a subtropical region of southern China: a 7-year survey. Eur J Clin Microbiol Infect Dis 2019; 38: 2355-2364.

20. Liu T, Li Z, Zhang S, Song S, Julong W, Lin Y, et al. Viral etiology of acute respiratory tract infections in hospitalized children and adults in Shandong province, China. Virol J 2015; 12: 168. 